\title{
Surgical correction of buried penis after traffic accident - a case report
}

\author{
Hiroshi Masuda, Haruhito Azuma*, Naoki Segawa, Yusaku Iwamoto, \\ Teruo Inamoto, Noboru Takasaki and Yoji Katsuoka
}

Address: Department of Urology Osaka Medical College, Takatsuki, Osaka 569-8686, Japan

Email: Hiroshi Masuda - m3255makiko@r2.dion.ne.jp; Haruhito Azuma* - uro004@poh.osaka-med.ac.jp;

Naoki Segawa -n146382@siz.saiseikai.or.jp; Yusaku Iwamoto - uro009@poh.osaka-med.ac.jp; Teruo Inamoto - pomodorijp@yahoo.co.jp;

Noboru Takasaki - info@midorigaoka.or.jp; Yoji Katsuoka - uro001@poh.osaka-med.ac.jp

* Corresponding author

Published: 08 June 2004

BMC Urology 2004, 4:6
Received: 0I February 2004

Accepted: 08 June 2004

This article is available from: http://www.biomedcentral.com/l47/-2490/4/6

(C) 2004 Masuda et al; licensee BioMed Central Ltd. This is an Open Access article: verbatim copying and redistribution of this article are permitted in all media for any purpose, provided this notice is preserved along with the article's original URL.

\begin{abstract}
Background: Buried penis, most commonly seen in children, is particularly debilitating in adults, resulting in inability to void while standing and it also affects vaginal penetration. We report a case of buried penis due to a traffic accident, which caused dislocation of the fractured pubic bone that shifted inside and pulled the penis by its suspensory ligament.
\end{abstract}

Case presentation: A 55-year-old man was admitted to our hospital with a chief complaint of hidden penis while in the sitting position. He had suffered a pelvic fracture in a traffic accident four years previously, and his penis was covered with suprapubic fat when he was in a sitting position. He was unable to have sexual intercourse. We performed a penile lengthening procedure, including inverse $\mathrm{V}$-Y-plasty of the dorsal skin of the penile root, suspensory desmotomy and fat removal, under general anesthesia. There was a good cosmetic result with satisfactory penile erection, which allowed successful sexual intercourse after surgery.

Conculsion: We performed penile elongation surgery with inverse V-Y-plasty of the dorsal skin of the penile root, suspensory desmotomy, and fat removal. Surgical treatment of buried penis achieves marked aesthetic and functional improvement, and benefits the majority of patients, resulting in satisfactory erection and successful sexual intercourse.

\section{Background}

Buried penis, most commonly seen in children, is particularly debilitating in adults, resulting in inability to void while standing and also affecting vaginal penetration. We report a case of buried penis due to a traffic accident, which caused dislocation of a fractured pubic bone that shifted inside and pulled the penis by its suspensory ligament. We performed desmotomy of the suspensory liga- ments as well as V-Y-plasty of the dorsal skin of the penis in order to release the penis from traction. These surgical procedures achieved a marked aesthetic and functional improvement, and benefited the patient, allowing satisfactory erection and successful sexual intercourse. 

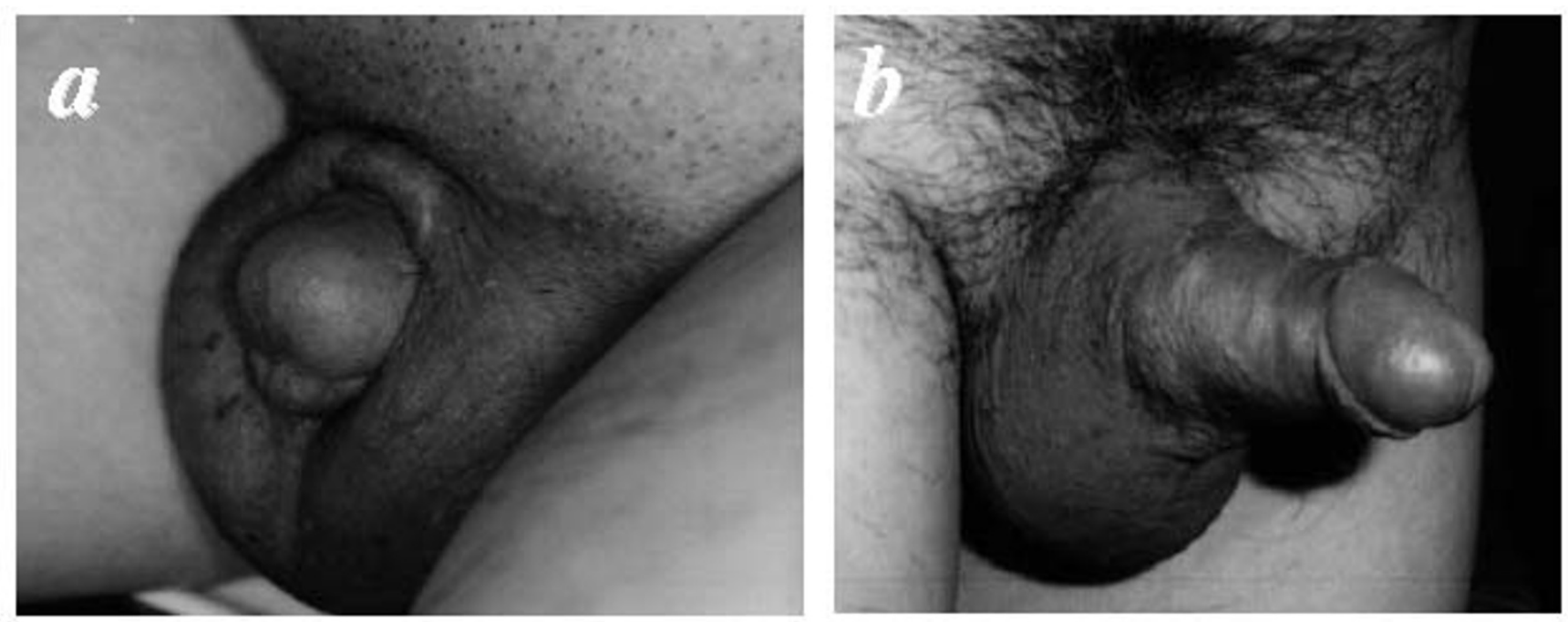

Figure I

Preoperative (a), and postoperative (b) appearance

\section{Case presentation}

A 55-year-old man was admitted to our hospital with a chief complaint of hidden penis in the sitting position (Fig. 1a). Four years previously, the patient had suffered a traffic accident, which had caused a dislocation fracture of the right hip joint and a fracture of the left pelvic bone. The dislocation fracture of the right hip joint was corrected promptly by surgical treatment, but the fracture of the left pelvic bone had been treated conservatively. The patient had medium height and build $(167 \mathrm{~cm} ; 60 \mathrm{~kg}$; BMI 1.628). Blood tests for endocrinological parameters were within the normal ranges, and he had not had any problem with sexual intercourse before the accident. At physical examination, we observed that his penis was completely covered with suprapubic fat, especially when the patient was sitting. Computed tomography revealed that the fractured left pubic bone had shifted inside (Fig. 2 ), pulling the penis by its suspensory ligament, and making it invisible when in a sitting position. The patient was unable to have sexual intercourse, and his small visible penis had caused severe loss of self-esteem. We performed a penile lengthening procedure with suspensory desmotomy, inverse V-Y-plasty of the dorsal skin of the penile root, and fat removal, under general anesthesia. After surgery, there was a good cosmetic result with satisfactory penile erection, which enabled successful sexual intercourse (Fig. 1b).

\section{Discussion}

This report highlights the evaluation and management principles in an unusual case of adult buried penis due to a traffic accident, and helps define the role of the plastic surgeon in such cases. Over the past 30 years there has been only one case report regarding buried penis due to a traffic accident [1]. Regarding the treatment of buried penis in adults, an understanding of the pathological anatomy and pathophysiology is extremely important [2$4]$. The penis is suspended from the pubis by the suspensory ligament, and remains fixed whereas the fat does not. In our patient, a traffic accident had caused dislocation of the pubic bone, which had shifted inside. The fractured pubis had pulled the penis by its suspensory ligament, and the penis had become buried in suprapubic fat, especially when the patient was in a sitting position. In this situation, the suspensory ligament gave no support to the penile root, but caused dislocation of the penile root due to traction. We therefore detached the suspensory ligament from the penile root in order to release the traction. We also performed fat removal with V-Y-plasty of the dorsal skin of the penile root for penile elongation surgery. Application of appropriate techniques for skin resurfacing depends on the degree of deficiency. Simple closure of the defect often suffices in patients with adequate skin. V-Y plasty will suffice in patients with moderate deficiency in the length of skin available [5]. In the present case, we performed fat removal with inverse V-Y-plasty of the dorsal skin of the penile root, and achieved a marked aesthetic and functional improvement with satisfactory erection and successful sexual intercourse. 


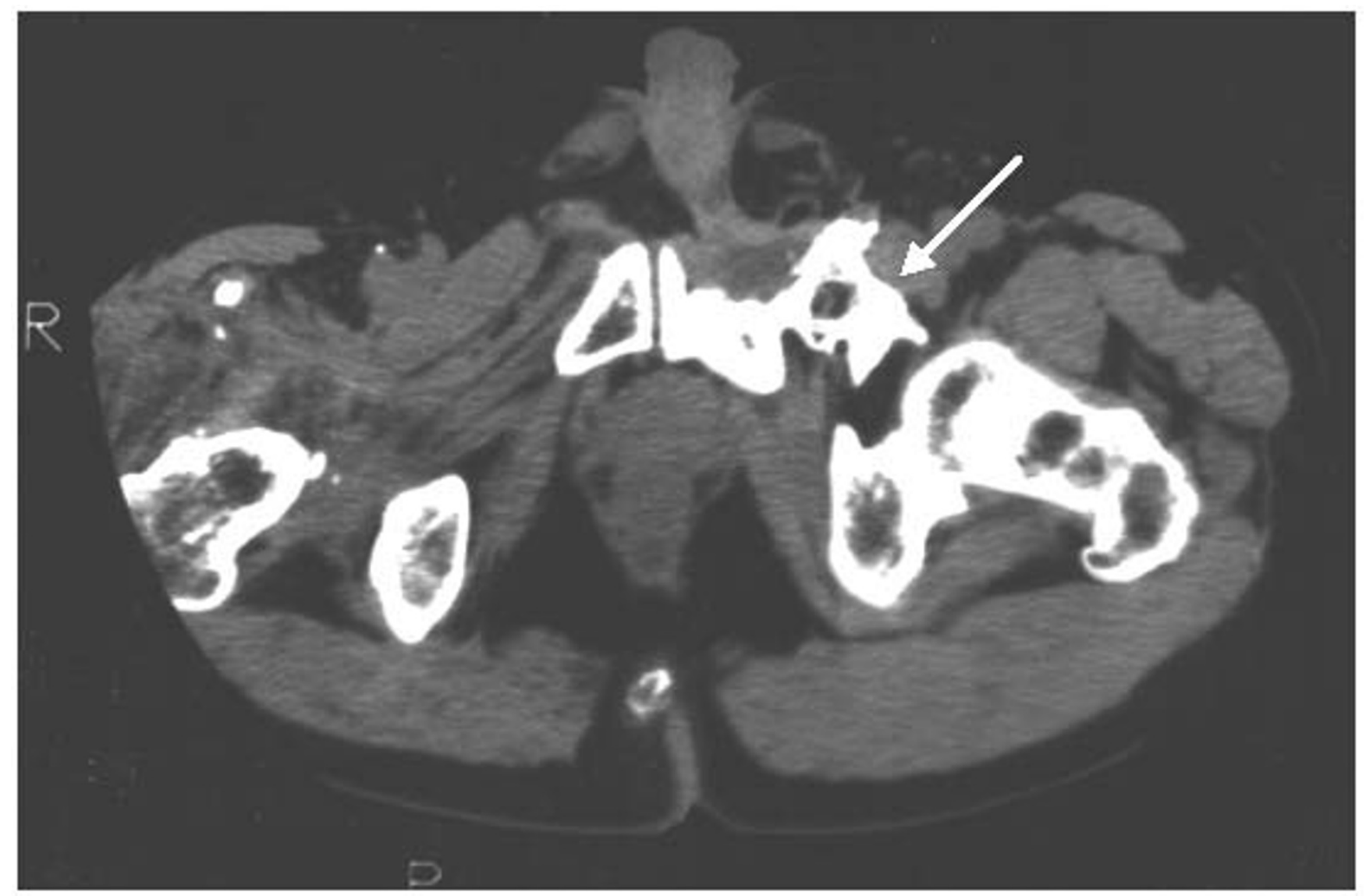

\section{Figure 2}

Computed tomography of the pelvis shows the fractured left pubic bone shifted to the left and head side (arrow).

\section{Conclusion}

Surgical approach for buried penis can achieve marked aesthetic and functional improvement, and benefits the patient resulting in satisfactory erection and successful sexual intercourse.

\section{References}

I. Simonnis LAJ, Borovets S, Van Driel MF, Ten Duis HJ, Mensink HJA: Erectile dysfunction due to a hidden penis after pelvic trauma. Int J Impot Res 1999, I I:53-55.

2. Donatucci CF, Ritter EF: Management of the buried penis in adult. J Urol 1998, 159:420-424.

3. Alici B, Culha M, Ozkara H, Akkus E, Hattat $\mathrm{H}$ : Management of buried penis in adults. Urol Int 1998, 61:183-5.

4. Chopra CW, Ayoub NT, Bromfield C, Witt PD: Surgical management of acquired (cicatricial) buried penis in an adult patient. Ann Plast Surg 2002, 49:545-9.

5. Wessells H, Lue TF, McAninch JW: Complications of penile lengthening and augmention seen I referral center. J Urol 1996, 155:1617-1620.

\section{Pre-publication history}

The pre-publication history for this paper can be accessed here: http://www.biomedcentral.com/1471-2490/4/6/prepub

Publish with Biomed Central and every scientist can read your work free of charge

"BioMed Central will be the most significant development for disseminating the results of biomedical research in our lifetime. "

Sir Paul Nurse, Cancer Research UK

Your research papers will be:

- available free of charge to the entire biomedical community

- peer reviewed and published immediately upon acceptance

- cited in PubMed and archived on PubMed Central

- yours - you keep the copyright
BiolMedcentral 